\title{
Synthesis of 3-alkoxypropan-1,2-diols from glycidol: experimental and theoretical studies for the optimization of the synthesis of glycerol derived green solvents
}

\author{
A. Leal-Duaso ${ }^{a, b}$, M. Caballero ${ }^{a}$, A. Urriolabeitia ${ }^{a}$, J. A. Mayoral ${ }^{a, b}$, J. I. García*b and E. Pires ${ }^{* a, b}$ \\ A straightforward synthetic methodology has been derived for the synthesis of glycerol monoethers from glycidol and \\ alcohols. Several homogeneous and heterogeneous basic catalysts have been tested, the best results being obtained with \\ readily available and inexpensive alkaline metal hydroxides. In the best cases, good yield of the desired monoether is \\ obtained in smooth reaction conditions, always with total conversion of glycidol. The selectivity of the reactions mainly \\ depends on the alcohol used, due to the concurrence of undesired side reactions. A mechanistic study, carried out through \\ computational DFT calculations, in which solvent effects are taken into account, also complemented with experiments, has \\ allowed to identify the main reaction paths taking place in reaction conditions, giving insights into the main causes affecting \\ the reaction selectivity and also into how it could be improved.
}

\section{Introduction}

Depletion of fossil fuels and raw materials has arisen a special interest in the development of new synthetic processes for the valorisation of renewables, vegetable oils being among the most widely used. Vegetable oil processing provides glycerol and fatty acids or esters, which have a huge number of applications. ${ }^{1-9}$ Valorisation of glycerol has become a trending topic due to the increasing of biodiesel production since the year 2000. Many efforts have been devoted to the synthesis of valuable products from this platform molecule, some of them displaying interesting uses as fuel additives, plasticizers, humectants, surfactants, etc. ${ }^{10-19}$ In this regard, the use of glycerol itself and its derivatives as solvents is a field of growing interest. ${ }^{20-25}$ Glycerol esters, ketals and ethers are commonly used as solvents and additives in cleaning, cosmetic, food and pharmaceutical industry. ${ }^{26-30}$

Glycerol ethers stand out among other glycerol derivatives by their relative chemical inertness, which make them very suitable as solvents. Apart from the synthesis of glycerol tertbutyl ethers from glycerol and isobutene, deeply studied due to their use as biofuel additives, ${ }^{31}$ the synthesis of alkyl glyceryl ethers from glycerol has been investigated in order to obtain a large class of compounds with tunable properties depending on the number and nature of the alkyl chains. Thus, recently,

\footnotetext{
a.Dr. E. Pires, Dr. J. A. Mayoral, M. Caballero, A. Urriolabeitia

Department of Organic Chemistry. Faculty of Science

University of Zaragoza

Calle Pedro Cerbuna, 12. E-50009 Zaragoza, Spain

E-mail:epires@unizar.esAddress here.

b.Dr. J. I. García, A. Leal-Duaso

Instituto de Síntesis Química y Catálisis Homogénea (ISQCH)

CSIC-University of Zaragoza

Calle Pedro Cerbuna, 12. E-50009 Zaragoza, Spain

E-mail: ji.garcia@csic.es

Electronic Supplementary Information (ESI) available: [details of any supplementary information available should be included here]. See DOI: 10.1039/x0xx00000x
}

Lemaire and co-workers have thoroughly reviewed the synthesis of glycerol ethers. ${ }^{32}$ These compounds can be synthesized either directly from glycerol ${ }^{33}$ or from industrial building blocks, such as glycidol, epichlorohydrin or 3chloropropane-1,2-diol, which can in turn be prepared from glycerol. ${ }^{32}$ The use of building blocks usually improves the selectivity of the process and allows to obtain mono-, di- and triethers with different alkyl chains in a controlled way. This variability in the nature of the substituents is important in order to prepare solvents with tunable properties, as described by García et al. ${ }^{34,35}$

Although there is a strong temptation to qualify glycerolderived solvents, and particularly glycerol ethers, as green, there are some aspects that must be correctly addressed in order to make this claim. One of them is related with physical properties. In this regard, glycerol ethers possess some favourable features, such as high boiling, flash points and low volatilities. Some of them, like the fluoroalkyl glycerol ethers, have special properties that made them suitable for the substitution of ionic liquids in some catalytic applications. ${ }^{36-38}$ Another key point is the toxicological profile of these compounds, an aspect that is currently under study in our group. ${ }^{39,40}$ Last, but not least, the synthetic procedures to obtain this family of glycerol derivatives should meet as much as possible the principles of Green Chemistry and, in order to be of practical applicability, they should also meet economic criteria. However, as mentioned by Lemaire and co-workers, ${ }^{32}$ despite the numerous potential applications of glycerol alkyl ethers, ${ }^{41-43}$ and especially of glycerol monoalkyl ethers (GMEs), scarce data on production as well as their demand can be found. This fact is probably due to the relatively recent interest in these products, on the one hand, as well as to the difficulty to make react selectively just one of the hydroxyl groups of glycerol, which are too similar in reactivity, on the other.

In this work we present a systematic study of the synthesis of GMEs starting from glycidol and several alcohols as reagents, 
without the need of an excess of glycidol, since this reagent is known to be toxic and irritant, ${ }^{44}$ and reasonably anticipated to be carcinogen for humans. ${ }^{45}$ Furthermore, the synthetic procedure does not require any additional solvent and uses readily available basic catalysts, with the aim to provide a greener, more effective synthetic methodology for the preparation of these compounds, compared to the syntheses previously described. ${ }^{46}$ The methodology proposed intends to be an alternative to previous or similar reported synthesis, in which metallic sodium has traditionally been used as a stoichiometric reagent to generate the alkoxide, and equimolecular amounts of salts are produced in the work up of the reaction. A greener synthetic procedure should reduce the amount of by-products and residues, improving both the atom economy and the $\mathrm{E}$ factor, not to mention the avoidance of using a dangerous reagent, such as metallic sodium. To this end, experimental studies have been complemented with computational mechanistic studies in order to rationalize the selectivity observed in the reactions.

\section{Results and discussion}

\section{Screening of the reaction conditions}

Previous works on the synthesis of GMEs described the use of sodium as starting reagent for the generation of the corresponding alkoxide and its subsequent reaction with glycidol 1 (Scheme 1). ${ }^{35}$ As it has been already commented in the introduction, GMEs are promising green solvents and the need of avoiding the use of hazardous reagents in their synthesis, such as metallic sodium, prompted us to do a screening of basic catalysts able to provide GME's (henceforth coded as [R.0.0]) in good yields, starting from glycidol $\mathbf{1}$ and different alcohols 2.

Both homogeneous catalysts, such as metal hydroxides or organic bases like 1,5,7-Triazabicyclo[4.4.0]dec-5-ene (TBD), and heterogeneous catalysts, such as TBD supported onto silica or polystyrene, the strongly basic resin Amberlyst ${ }^{\circledR}$ IRA 400 or mixed oxides, have been tested.

For the initial optimization of the reaction conditions, the benchmark reaction between glycidol $\mathbf{1}$ and methanol $\mathbf{2 a}$, catalyzed by $\mathrm{KOH}$, was studied.

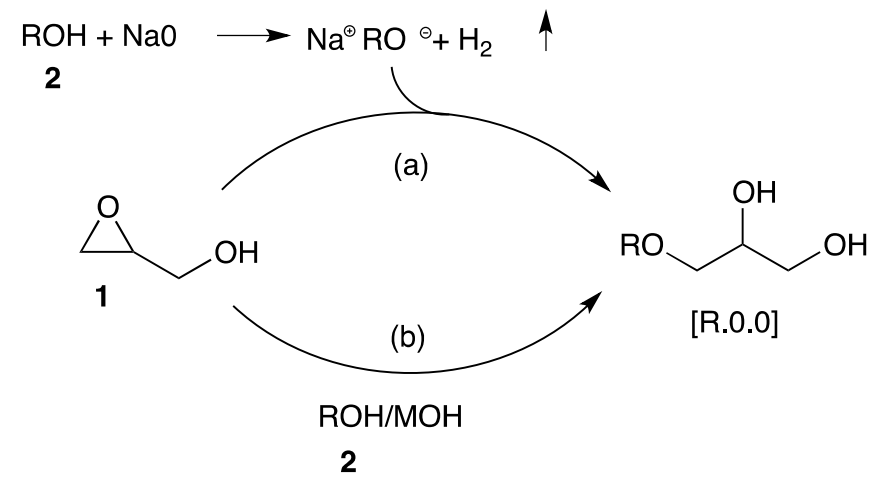

Scheme 1. Synthesis of glycerol monoethers [R.0.0] from glycidol 1 and alcohols 2. a) Synthesis previously reported, ${ }^{35}$ b) This work.
The influence of the reaction temperature, catalyst molar proportion, reagents concentration and speed of glycidol addition were the parameters considered.

First, the optimization of the amount of catalyst was done. Reactions with $10 \%, 20 \%$ and $30 \%$ mol of $\mathrm{KOH}$ were carried out at 65 으 (the refluxing temperature of methanol). Total conversions of glycidol 1 were achieved in $120 \mathrm{~min}$ both with $20 \%$ and $30 \%$ mol catalyst (Figure 1 ), and up to $93 \%$ yield of $3-$ methoxypropane-1,2-diol (henceforth [1.0.0]) was obtained. When using $10 \%$ mol catalyst lower yields and conversions were observed at the same reaction time. As mentioned in the Introduction, given the known issues with the use of glycidol, ${ }^{32,44}$ it is important that the synthetic procedure assures its total conversion, thus avoiding its presence during the product purification steps, as well as in the reaction waste. From this preliminary study, $20 \% \mathrm{~mol}$ seems to be the most adequate catalyst proportion for this reaction.

Next, the effect of reaction temperature was studied using $20 \% \mathrm{~mol} \mathrm{KOH}$. Three reaction temperatures were considered: 65,45 , and 25 으. A decrease both in the conversion of glycidol 1 and in the yield of the desired product was observed with the decrease of temperature (Table 1). Furthermore, the selectivity to the desired product also seems to decrease with the reaction temperature.

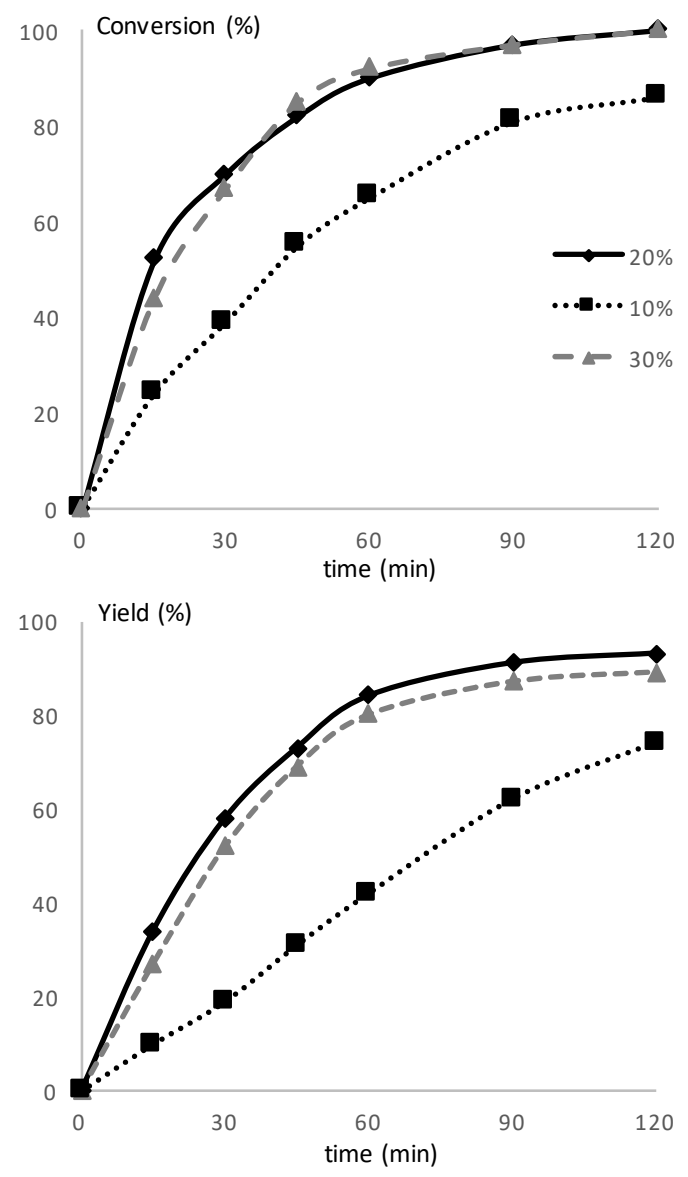

Figure 1. Optimization of the amount of catalyst in the reaction of glycidol 1 with methanol 2a, catalyzed by $\mathrm{KOH}$. 
Table 1. Effect of the temperature in the reaction of glycidol 1 with methanol $2 \mathrm{a}$, catalyzed by $\mathrm{KOH}(20 \% \mathrm{~mol})$. [a

\begin{tabular}{|c|c|c|c|c|c|c|}
\hline \multirow[b]{2}{*}{ Time (min) } & \multicolumn{3}{|c|}{ Conversion (\%) } & \multicolumn{3}{|c|}{ Yield (Selectivity) (\%) } \\
\hline & $65 \stackrel{\circ}{\circ}$ & $45 \stackrel{\circ}{\circ}$ & $25^{\circ} \mathrm{C}$ & $65 \stackrel{\circ}{\circ}$ & $45 \stackrel{\circ}{C}$ & $25 \stackrel{\circ}{\circ}$ \\
\hline 15 & 52 & 41 & 29 & $34(65)$ & $31(76)$ & $21(72)$ \\
\hline 30 & 70 & 59 & 45 & $58(83)$ & $50(85)$ & $37(82)$ \\
\hline 45 & 82 & 71 & 58 & $73(89)$ & $63(89)$ & $49(85)$ \\
\hline 60 & 90 & 80 & 67 & $84(93)$ & 71(89) & $58(86)$ \\
\hline 90 & 97 & 89 & 78 & $91(93)$ & $82(92)$ & $67(86)$ \\
\hline 120 & 100 & 94 & 84 & $93(93)$ & $89(95)$ & $74(88)$ \\
\hline
\end{tabular}

[a] Reaction conditions: 15:1 molar ratio of methanol 2a to glycidol $\mathbf{1}$ and slow addition of glycidol 1 to the reaction (15 $\mathrm{min}$ ).

On the other hand, this selectivity is not constant along the reaction time, reaching a maximum at the end of the reaction. We will return on this point in the mechanistic discussion. After these series of experiments, $65 \stackrel{\circ}{\circ}$ was therefore chosen as the standard reaction temperature.

An effect of the reagents concentration was also observed in the synthesis of [1.0.0]. In an attempt of reducing the excess of methanol $\mathbf{2 a}$ from 15 to $7.5 \mathrm{~mol}$ per mol of glycidol $\mathbf{1}$ (but at the expense of significantly reducing the total reaction volume), a rise in the conversion of glycidol was observed. Total conversion was achieved in 90 min reaction time. Nevertheless, the selectivity towards the desired product [1.0.0] was reduced from $93 \%$ to $83 \%$, and the formation of a higher amount of byproducts was observed by GC.

The effect of the way in which glycidol $\mathbf{1}$ was added to the reaction medium was also studied (Scheme 2 ). Adding glycidol 1 in a dropwise manner strongly improved the selectivity of the reaction from $70 \%$ when glycidol 1 was added at once, to $93 \%$ when it was slowly added. This fact is most probably due to the inhibition of glycidol self-reaction, although other competing pathways cannot be excluded, as it will be discussed below.

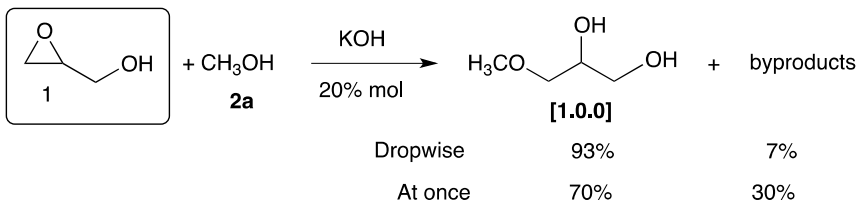

Scheme 2. Study of the influence of the addition of glycidol 1 to the reaction media on the reaction selectivity

Finally, for the sake of comparison, a reaction was carried out without a catalyst, otherwise in the same reaction conditions. In this case, $68 \%$ conversion of glycidol 1 was observed at $48 \mathrm{~h}$ reaction time, with $38 \%$ yield of [1.0.0] $(56 \%$ selectivity). After $2 \mathrm{~h}$ reaction time, conversion was only $15 \%$, with $5 \%$ yield of [1.0.0] (33\% selectivity). Therefore, although the uncatalyzed reaction may take place, a strong interference in the results of the catalyzed processes is not to be expected in most cases.

To summarize this section and to proceed with the study of several basic catalysts with different alcohols, the standard reaction conditions finally chosen were 65 oc reaction temperature, using $20 \%$ mol catalyst, 15:1 molar ratio of alcohol to glycidol and slow addition of glycidol to the reaction medium (15 min, using a syringe pump).

\section{Scope of the synthesis with different bases and alcohols}

Several metal hydroxides were first tested as homogeneous catalysts for the synthesis of GMEs [R.0.0], namely $\mathrm{LiOH}, \mathrm{NaOH}$, $\mathrm{KOH}$ and $\mathrm{Sr}(\mathrm{OH})_{2}$. Besides methanol $2 \mathrm{a}$, a set of five alcohols was selected for the synthesis of [R.0.0] derivatives bearing different alkyl moieties. Thus, alcohols with linear alkyl chains (methanol 2a, ethanol $\mathbf{2 b}$ and butanol $\mathbf{2 c}$ ), branched chains (isopropanol 2d and 2-ethylhexan-1-ol 2e), fluorinated chains (2,2,2-trifluoroethanol $\mathbf{2 f}$ ) and aromatics (phenol $\mathbf{2 g}$ ) were selected to cover a broad spectrum of properties. These alcohols are not only expected to display different reactivity, due to electronic and steric reasons, but, as far as they are also used as solvents, they are also able to modify to some extent the polarity of the reaction medium. Selection of these alcohols was done both due to their different structures, as well as for the interest of the GMEs obtained. Thus, fluorinated glycerol derivatives, such as the closely related 1,3-bis $(2,2,2-$ trifluoroethoxy)propan-2-ol [3F.0.3F], have been successfully used as reaction media in epoxidation and enzymatic reactions. ${ }^{38,47-49}$ 3-(2-ethyl)hexyl-oxypropane-1,2-diol [6(2).0.0] is a frequent compound in cosmetic formulations. ${ }^{32}$ Finally, glyceryl monoaryl ethers are also used in pharmaceutic and cosmetic formulations, as well as intermediates in the synthesis of drugs and pharmaceuticals. ${ }^{50-52}$

The main results of these experiments are gathered in Table 2. When using methanol 2 (Table 2 , entry 1 ), total conversions, good yields of the desired product are obtained. $\mathrm{NaOH}$ and $\mathrm{KOH}$ are the preferred catalysts in this case, since better yields of the desired product are achieved.

When increasing the length of the alkyl chain of the alcohol (EtOH 2b, BuOH 2c) (Table 2, entries 2, 3), total conversions of glycidol $\mathbf{1}$ are still obtained in $2 \mathrm{~h}$ reaction time, but the selectivity of the reaction toward the desired products is significantly reduced. In this case, the formation of heavier compounds, involving dimerization of glycidol in one or another way, was observed by gas chromatography/mass spectrometry. This fact proves the existence of competitive side reactions, which will be discussed in the mechanistic study section. This effect is even more dramatic when using secondary and less active alcohols, such as isopropanol $\mathbf{2 d}$ or 2-ethylhexan-1-ol $\mathbf{2 e}$ (Table 2, entries 4, 5). In all cases, $\mathrm{NaOH}$ and $\mathrm{KOH}$ seem to be more suitable as catalysts, as better yields of the target [R.0.0] are obtained. 
Table 2. Conversions and yields in the reaction of glycidol 1 with different alcohols $\mathbf{2}$, catalyzed by metal hydroxides. [a]

\begin{tabular}{|c|c|c|c|c|c|c|c|c|c|c|c|}
\hline \multirow[b]{2}{*}{ Entry } & & \multirow[b]{2}{*}{ Alcohol } & \multirow[b]{2}{*}{ product } & \multicolumn{2}{|c|}{$\mathrm{LiOH}$} & \multicolumn{2}{|c|}{$\mathrm{NaOH}$} & \multicolumn{2}{|c|}{$\mathrm{KOH}$} & \multicolumn{2}{|c|}{$\mathrm{Sr}(\mathrm{OH})_{2}$} \\
\hline & & & & $\begin{array}{c}\text { Conv. } \\
(\%)\end{array}$ & $\begin{array}{c}\text { Yield (Sel.) } \\
(\%)\end{array}$ & $\begin{array}{c}\text { Conv. } \\
(\%)\end{array}$ & $\begin{array}{c}\text { Yield (Sel.) } \\
(\%)\end{array}$ & $\begin{array}{c}\text { Conv. } \\
(\%)\end{array}$ & $\begin{array}{l}\text { Yield (Sel.) } \\
(\%)\end{array}$ & $\begin{array}{c}\text { Conv. } \\
(\%)\end{array}$ & $\begin{array}{c}\text { Yield (Sel.) } \\
(\%)\end{array}$ \\
\hline 1 & $2 a$ & $\mathrm{MeOH}$ & {$[1.0 .0]$} & 100 & $86(86)$ & 100 & 92(92) & 100 & 93(93) & 100 & $73(73)$ \\
\hline 2 & $2 b$ & $\mathrm{EtOH}$ & {$[2.0 .0]$} & 99 & $47(47)$ & 100 & $54(54)$ & 100 & $57(57)$ & 83 & $29(35)$ \\
\hline 3 & 2c & $\mathrm{BuOH}$ & {$[4.0 .0]$} & 100 & $48(48)$ & 100 & $58(58)$ & 100 & $60(60)$ & 100 & $42(42)$ \\
\hline 4 & $2 d$ & iPrOH & [3i.0.0] & 86 & $20(23)$ & 100 & $27(27)$ & 100 & $30(30)$ & 56 & $4(7)$ \\
\hline 5 & $2 e$ & 2-ethylhexan-1-ol & {$[6(2) .0 .0]$} & 80 & $17(21)$ & 100 & $27(27)$ & 100 & $36(36)$ & 55 & $3(5)$ \\
\hline 6 & $2 f$ & Trifluoroethanol & {$[3 F .0 .0]$} & 91 & $89(98)$ & 46 & $36(78)$ & 44 & $35(80)$ & 53 & $42(79)$ \\
\hline 7 & $2 g$ & Phenol & [Ph.0.0] & 92 & $63(68)$ & 66 & $48(73)$ & 46 & $22(48)$ & 83 & $63(76)$ \\
\hline
\end{tabular}

[a] Reaction conditions: $20 \%$ mol catalyst, $65 \stackrel{\circ}{\circ}, 15: 1$ molar ratio of alcohol $\mathbf{2}$ to glycidol 1 , with slow addition of glycidol 1 to the reaction (15 min) and $2 \mathrm{~h}$ reaction time.

Finally, when using more acidic alcohols, such as 2,2,2trifluoroethanol $2 \mathrm{f}$ (TFE) (Table 2 entry 6), better selectivities are observed in most cases, compared to the ones obtained with ethanol $\mathbf{2 b}$ (Table 2 entry 2 ), although the reactions are noticeably slower. This fact seems to indicate that the relative acidity of the starting alcohol vs. the acidities of glycidol and the [R.0.0] product is a determinant factor for the selectivity observed in these reactions, most probably because of the inhibition of the formation of the corresponding glycidol and [R.0.0] alkoxides (see below).

In the case of TFE $\mathbf{2 f}$, $\mathrm{LiOH}$ appears to be the best catalyst, with $91 \%$ conversion in $2 \mathrm{~h}$ (with an excellent $98 \%$ selectivity to the corresponding [3F.0.0] product), which can be pushed up to $100 \%$ if the reaction time is increased up to $6 \mathrm{~h}$. Similar trends are observed when using another acidic alcohol like phenol $2 \mathrm{~g}$ (Table 2, entry 7). To summarize, these series of experiments show that $\mathrm{NaOH}$ and, above all, $\mathrm{KOH}$, are the best catalysts in the case of alkyl alcohols. In all cases, $100 \%$ conversion of glycidol is reached at $2 \mathrm{~h}$ reaction time. However, the selectivity to the target [R.0.0] product is strongly dependent on the alcohol. On the other hand, $\mathrm{LiOH}$ is a better catalyst for more acidic alcohols, whose corresponding alkoxides are less nucleophilic and hence less reactive.

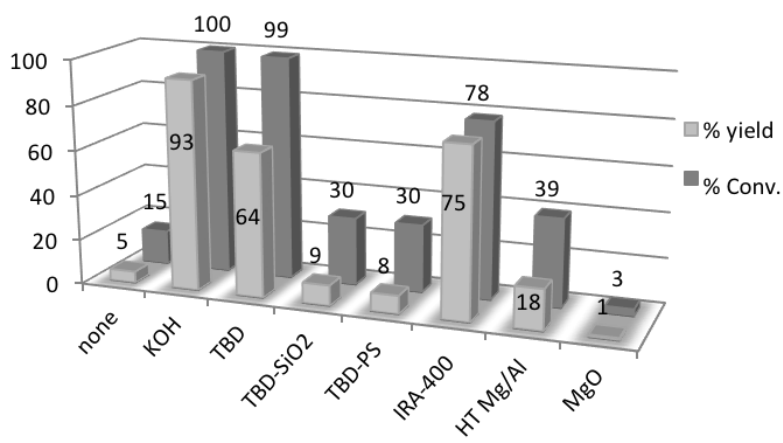

Figure 2. Comparison of the results of the reaction of glycidol 1 with methanol $2 \mathrm{a}$, catalyzed by different basic solids. Reaction conditions: $20 \%$ mol catalyst, 65 oC reaction temperature and $2 \mathrm{~h}$ reaction time.

Unfortunately, the majority of the heterogeneous catalysts tested gave poor results both in glycidol conversion and product yield. Only Amberlyst ${ }^{\circledR}$ IRA-400 led to acceptable results. In this case, recycle and reuse of the catalyst was studied in order to know if it could be considered as an efficient and affordable catalyst for the synthesis of [1.0.0]. Results showed (Figure 3 ) that total conversions of glycidol can be achieved, keeping an excellent $96 \%$ selectivity to [1.0.0], but at the expense of increasing the reaction time. Unfortunately, the yield of [1.0.0] decreased in each run. In an attempt to recover the activity of the catalyst, a sample of solid catalyst used in three runs was treated with a solution of $\mathrm{KOH}$ in methanol and then thoroughly washed with methanol. In this case, the activity of the catalyst was partially recovered and the yield was increased from $15 \%$ to $58 \%$ at $2 \mathrm{~h}$ reaction time, with $81 \%$ selectivity, which further increased up to $85 \%$ after $24 \mathrm{~h}$ reaction time.

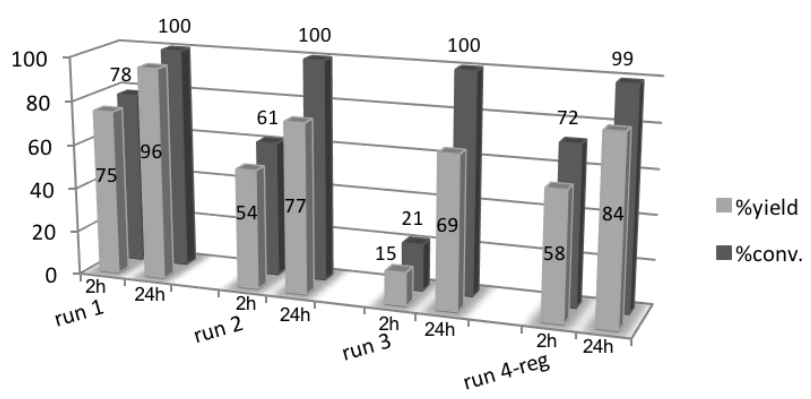

Figure 3. Results of the reuse of Amberlyst ${ }^{\circ}$ IRA-400 in the reaction of glycidol 1 with methanol $2 \mathrm{a}$ at $2 \mathrm{~h}$ and $24 \mathrm{~h}$ reaction time.

Amberlyst ${ }^{\circledast}$ IRA-400 was also tested as catalyst in the reactions with the selected set of alcohols (Figure 4). Results with $\mathrm{KOH}$ are included for the sake of comparison. Except in the case of methanol $\mathbf{2 a}$, yields of the [R.0.0] products are always lower than those obtained using $\mathrm{KOH}$ or $\mathrm{NaOH}$ as catalysts

Overall, Amberlyst ${ }^{\circledR}$ IRA-400 can be considered a good catalyst for the synthesis of [1.0.0], with a total turnover number of 12.1 in three consecutive runs. However, it is worth mentioning that the price of the catalyst, together with the big amount needed $(71 \% \mathrm{w} / \mathrm{w}$ with respect to glycidol), are strong difficulties to overcome when scaling up the synthesis of this glycerol ether. For the rest of alcohols, the activity of Amberlyst ${ }^{\circledast}$ IRA-400 is too low to be considered for large scale syntheses of GMEs. 


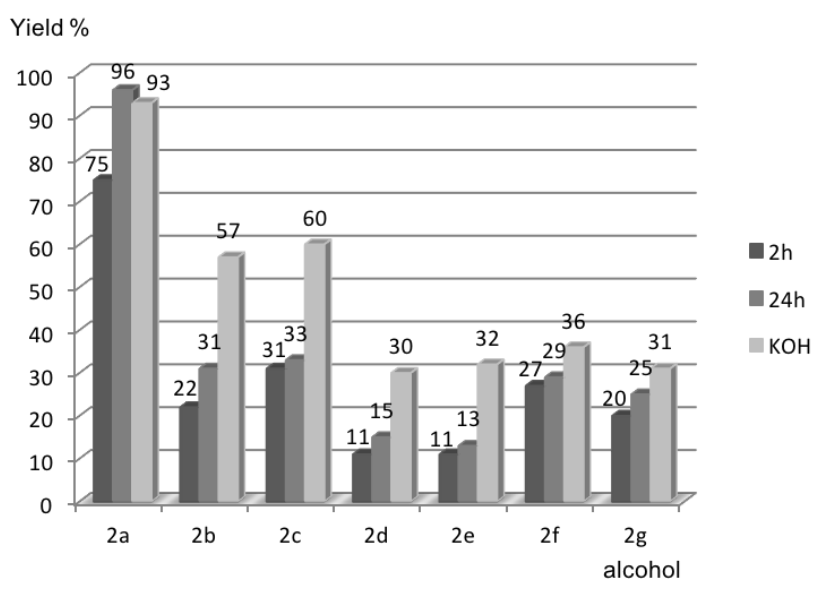

Figure 4. Yields of [R.0.0] in the reaction catalyzed by Amberlyst ${ }^{\circledR}$ IRA-400. Results at $2 \mathrm{~h}$ and $24 \mathrm{~h}$ reaction time. Results with $K O H$ at $2 \mathrm{~h}$ reaction time (right column) are also included for comparison.

\section{Mechanistic considerations}

The mechanistic study of the reaction of alkoxides with glycidol is a complex task, given that both glycidol and reaction products are alcohols, so they may participate in side reactions. In particular, glycidol has a $\mathrm{pK}_{\mathrm{a}}$ lower than those of the most common alkyl alcohols, and therefore it tends to deprotonate in reaction conditions, favouring self-reaction, except in the case of the most acidic alcohols like TFE $\mathbf{2 f}$ or phenol $\mathbf{2 g}$. On the other hand, the alkoxide formation from a metal hydroxide and an alcohol leads to the concomitant formation of a water molecule, which may also participate in undesired side reactions (for instance, the formation of glycerol). Figure 5 displays these possibilities, organized as catalytic cycles.

Only cycle I leads to the desired [R.0.0] product (8), whereas the rest correspond to side reactions. Cycles II and III represent the subsequent reaction of [R.0.0] with a second glycidol molecule through either the primary or secondary hydroxyl group, leading to products $\mathbf{1 1}$ and $\mathbf{1 4}$, respectively. Cycle IV represents the dimerization reaction of glycidol (1), leading to an epoxide intermediate (18), susceptible to react with a molecule of alkoxide (3) to lead to $\mathbf{1 1}$ (cycle V). Finally, water may participate in epoxide hydrolysis processes, leading either to glycerol (26) (cycle VII) or to a diglycerol (23) (cycle VI).

$$
[\mathrm{RO} \underbrace{\mathrm{O} \mathrm{j}^{-\mathrm{H}}}_{6}]^{\ddagger}
$$

a: $\mathrm{R}=\mathrm{Me}$

b: $\mathrm{R}=\mathrm{Bu}$

c: $\mathrm{R}=\mathrm{CF}_{3} \mathrm{CH}_{2}$
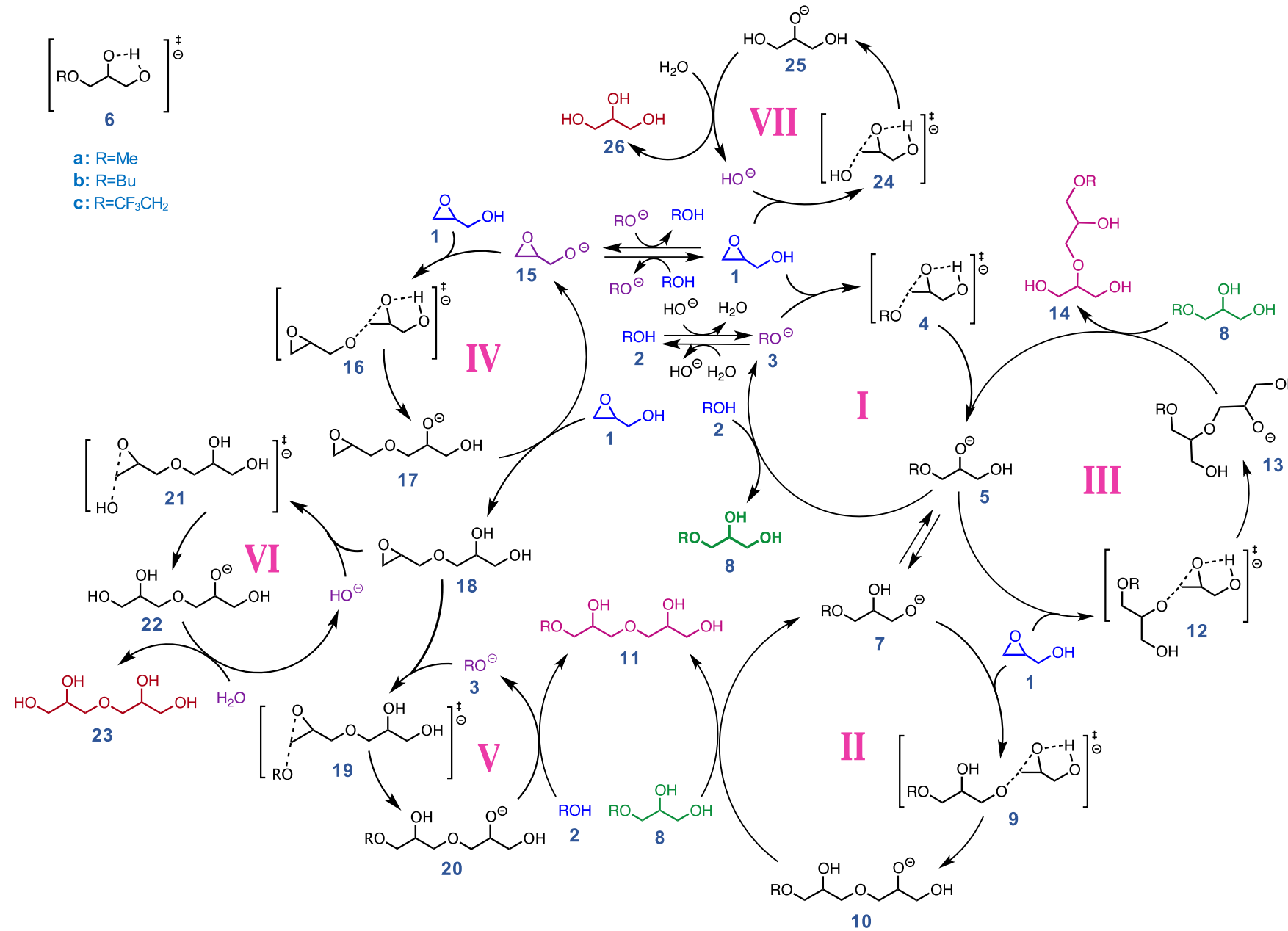

Figure 5. Possible catalytic cycles involved in the reaction of glycidol 1 with alcohols 2 , catalyzed by bases. 
Of course, there are many other possibilities, but we only intend to show those most probably involved in the reaction progress. It is important to note that glycidol is a chiral molecule and in this work it has been used in racemic form, so all the dimerization reactions lead to the formation of two pairs of diastereomers. This circumstance has also been taken into account in the computational study. Only the lowest energy paths will be discussed here, but complete information about all the possible reaction paths is included in the Supporting Information.

All the reagents, intermediates, transition structures and products in the cycles shown in Figure 5 have been calculated at the PCM(solvent)/B3LYP/6-311++G(d,p) theoretical level for two alcohols: methanol (2a) and butanol (2c). These alcohols were chosen because of the different selectivities to the corresponding [R.0.0] ether experimentally observed. A partial computational exploration of cycles I and IV was also carried out for 2,2,2-trifluoroethanol (TFE) (2f). In each case, solvent effects were taken into account through the PCM self-consistent reaction field model, using the internal parameterization included in the Gaussian 09 program for the aforementioned solvents. ${ }^{53}$ Complete details concerning molecular coordinates and energies of the calculated structures are available in the Supporting Information.

The first point to be discussed is the reaction of formation of the alkoxide from the alcohol and the metal hydroxide. This process takes place before adding the second reagent (glycidol) and is an equilibrium process. The equilibrium constants calculated for methanol (2a) and butanol (2c), based on Scheme 3 , are 1.16 and 0.68 , respectively.

$$
\mathrm{ROH}+\mathrm{HO}^{\ominus} \stackrel{\mathrm{Keq}}{\rightleftharpoons} \mathrm{RO}{ }^{\ominus}+\mathrm{H}_{2} \mathrm{O}
$$

Scheme 3. Alcohol deprotonation equilibrium in the formation of the alkoxides

However, as the alcohol is in a huge molar excess in reaction conditions (75:1 with regard to the base), both equilibria are completely shifted toward the formation of the corresponding alkoxide, so less than $2 \% \mathrm{~mol}$ of free hydroxide (HO-) is present at equilibrium. The case of TFE (2f) is even clearer, since the calculated equilibrium constant increases up to $1.85 \cdot 1011$. In these conditions, cycles VI and VII should play a minor role, given the residual concentration of $\mathrm{HO}-$ and the calculated activation barriers, not especially low. From the experimental point of view, glycerol (26) is not detected in significant amounts in reaction conditions, whereas diglycerol (23) is detected as an impurity present in commercial glycidol and its concentration increases only marginally during the reaction. For these reasons, the mechanistic discussion will be centred on the rest of the cycles involving the main reagents and intermediates

The reaction profiles of cycles $\mathbf{I}-\mathbf{V}$ for both methanol $2 \mathrm{a}$ and butanol $2 \mathrm{c}$ are displayed in Figure 6 . Concerning methanol, it can be realized that the reaction of methoxide (3a) with glycidol (1) (cycle I) has an intrinsic activation barrier lower than that of the dimerization of glycidol, occurring through the reaction of 1 with oxiran-2-ylmethoxide (15). However, it must be pointed out that in reaction conditions $\mathbf{1}$ is easily deprotonated to give 15 (the calculated equilibrium constant at $65{ }^{\circ} \mathrm{C}$ is 84 ). In these Curtin-Hammett conditions the effective activation barrier for the first reaction in cycle I turns to be higher by $1.8 \mathrm{kcal} \mathrm{mol}^{-1}$
(Figure 6a). In the case of butanol (Figure 6b), the situation is even worse, since the glycidol deprotonation equilibrium has a higher equilibrium constant (185), and the first step of cycle $\mathbf{I}$ is disfavoured by $3.8 \mathrm{kcal} \mathrm{mol}^{-1}$. This means that the reaction of dimerization of glycidol is intrinsically favoured over its reaction with alkoxides, which explains the difficulty to obtain good selectivities to the desired [R.0.0] products (8).

However, computational mechanistic studies often give a partial view of the reaction complexity, since they deal exclusively with energy landscapes (the so-called Erepresentation, following the Kozuch's and Shaik's definition $)^{54}$ and neglect concentration effects. In our case, the latter are not negligible at all, since the alcohol is in a large excess with regard to both glycidol (1) and its deprotonated form (15). In order to get an insight into the importance of concentration effects, we carried out a numerical simulation of the reaction kinetics on a simplified system, in which only cycles I and IV were taken into account. Rate constants were estimated from the activation barriers shown in Figure 6 using equation (1).

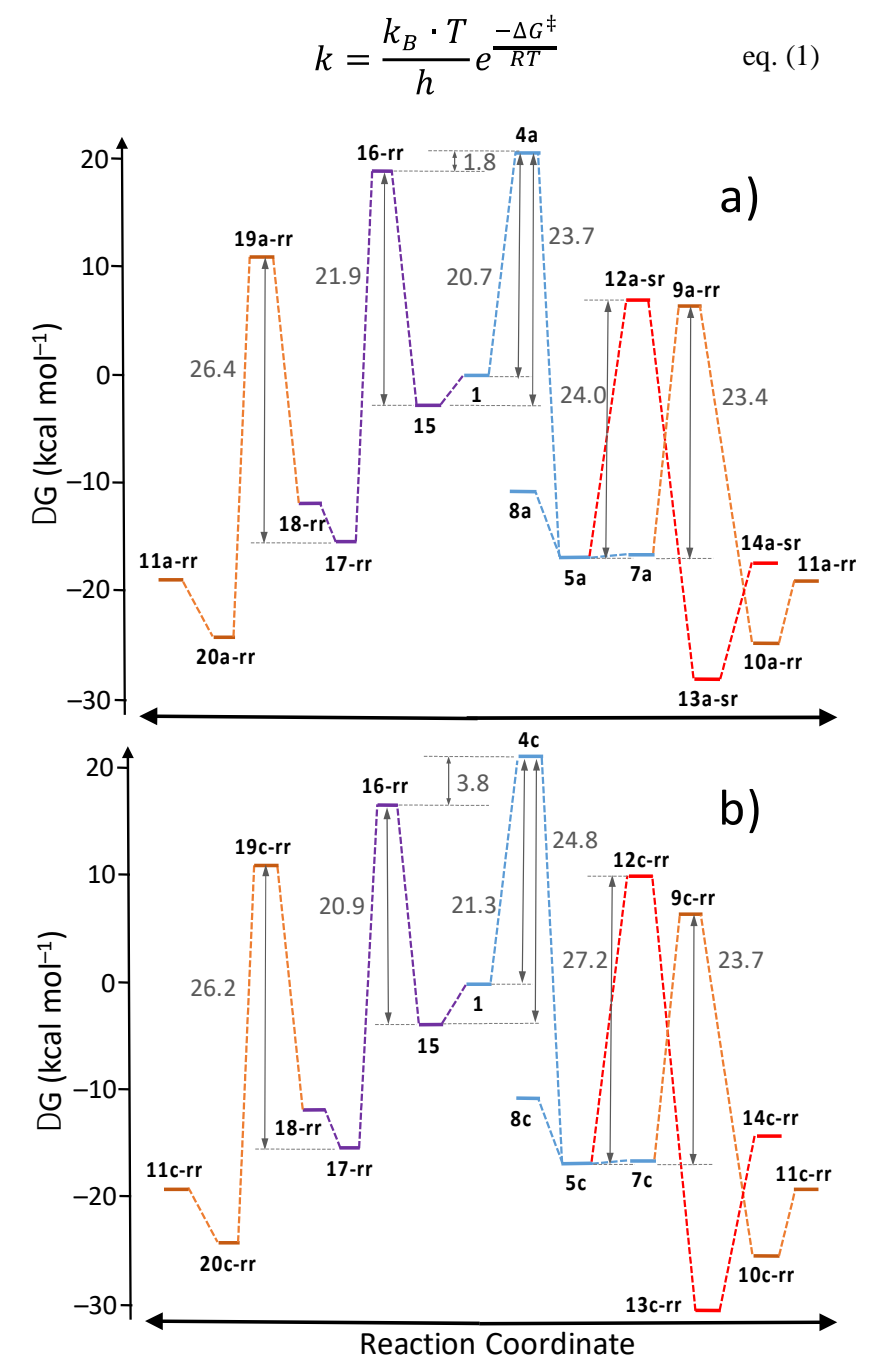

Figure 6. Calculated reaction coordinate profiles of the different reaction pathways for the reactions of glycidol 1 with methanol $2 \mathrm{a}$ (a) and butanol $2 \mathrm{c}$ (b). 
Protonation-deprotonation equilibria were also considered, using the corresponding calculated $\mathrm{K}_{\text {eq }}$ in each case. Initial concentrations were set to reproduce the experimental conditions used. Further details on these simulations can be found in the Supporting Information.
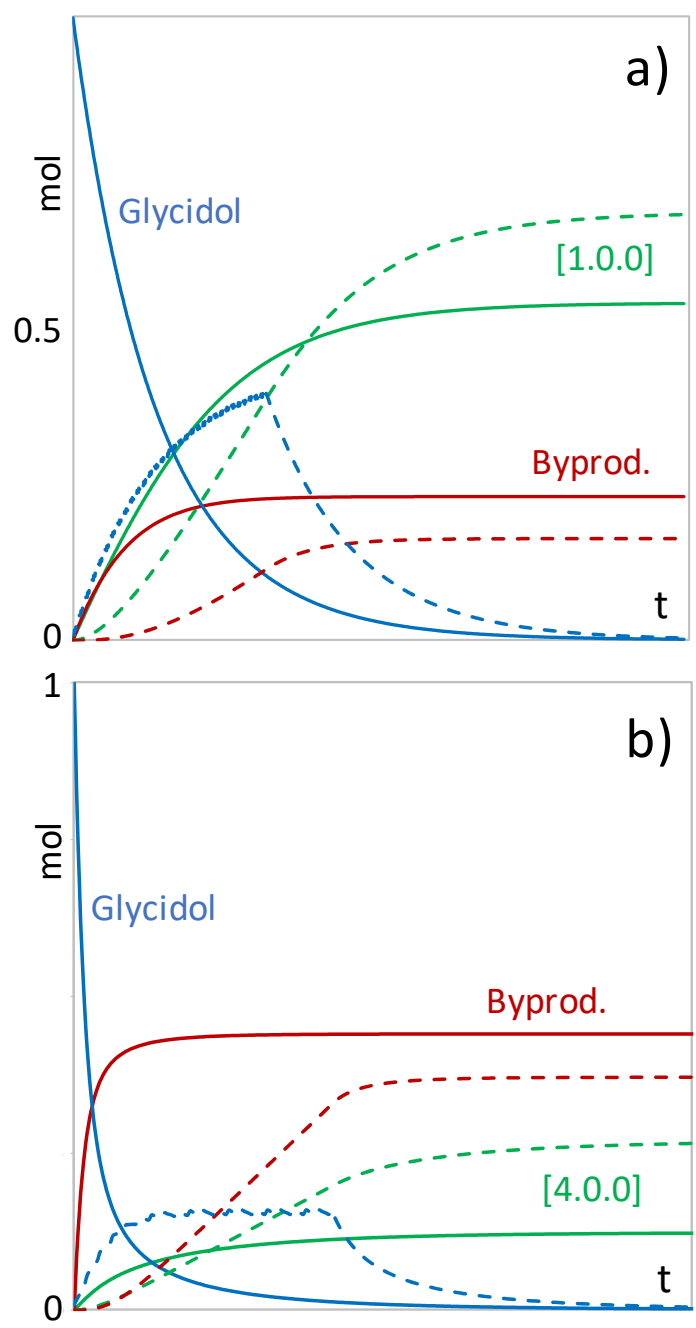

Figure 7. Simulations of the kinetics for the reactions of glycidol 1 with methano 2a (a) and butanol 2c (b). Continuous lines represent glycidol addition at once, and dashed lines, dropwise glycidol addition

Figure 7a displays the time evolution of the concentration of glycidol (1), [1.0.0] (8a), and glycidol dimer (18). Continuous lines represent the kinetics in the case of a reaction in which all the glycidol is added at once at the beginning. Dashed lines represent the kinetics when glycidol is added dropwise during the first minutes of the reaction. When glycidol is added at once, there is a smooth decrease until complete disappearance. Interestingly, the selectivity to the desired [1.0.0] (8a) product (54\% regarding the initial amount of glycidol) is higher than that to glycidol dimeric products, in spite of the higher activation barrier of the former process, which illustrates the importance of concentration effects. When $\mathbf{1}$ is added dropwise, the selectivity to 8 a further increases up to $68 \%$ as expected, since the principal side reaction is order 2 in glycidol.
Regarding the reactions with butanol, the time evolution of the concentrations of $\mathbf{1 , ~ 8 c}$ and $\mathbf{1 8}$ are shown in Figure $\mathbf{7 b}$. In this case, the higher barrier difference between both reaction paths results in a lesser influence of reagents concentrations. As can be seen, when $\mathbf{1}$ is added at once the selectivity to the desired product [4.0.0] (8c) is as low as $12 \%$, glycidol dimerization being largely favoured. When $\mathbf{1}$ is added dropwise, the selectivity to $8 \mathrm{c}$ more than doubles up to $27 \%$, but dimerization is still the most favoured process. As happened in the preceding case, the results experimentally obtained are far better than these ( $42 \%$ selectivity for batch conditions and $60 \%$ for dropwise addition), which is undoubtedly due to the rough approximation made to estimate the rate constants values from the calculated activation barriers. Nevertheless, the kinetic simulations show the same qualitative trend observed in the experiments, thus confirming the validity of the mechanistic proposal. It is worth noting that in butanol, the computational study reveals that the glycidol dimerization process is faster, with regard to what happens in methanol (calculated activation barriers are 20.9 and $21.9 \mathrm{kcal} \mathrm{mol}^{-1}$, respectively), which is also in line with the experimental conversions observed, both in at once and in dropwise addition conditions (see Tables S1, S2 in the Supporting Information). Of course, the existence of other reaction pathways must result in a much more complex product distribution. It is important to note that the protonation equilibria of intermediates $\mathbf{5}$ and $\mathbf{7}$ to the desired products 8 is thermodynamically disfavoured (Figure 6). This fact favours the further reaction of these intermediates (principally 7 , due to the lower activation barriers calculated for this step) with a second glycidol molecule in cycles III and II, respectively. The situation again is worse in the case of butanol reactions, given that the effective activation barrier leading to by-product 11c-rr (cycle II) is even lower than that of the initial reaction (cycle I) (23.7 vs. $24.8 \mathrm{kcal} \mathrm{mol}^{-1}$ ), which contributes to a lower selectivity to $8 \mathrm{c}$.

The feasibility of these competing pathways was experimentally tested in the case of the reaction of ethanol, which displays a behaviour almost identical to that of butanol, but is easier to analyse by GC and NMR. When the 3ethoxypropan-1,2-diol [2.0.0] was made react with glycidol, complete disappearance of the latter was observed after a short reaction time and $\mathbf{1 1}$ was observed and identified by NMR as the major product. No significant amount of $\mathbf{1 4}$ could be spotted in the reaction analysis, which is in agreement which the higher activation barrier for the pathway leading to this product (3.5

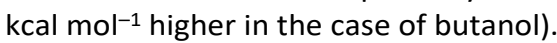

Concerning the reactions with more acidic alcohols, the theoretical calculations carried out for TFE (2f) are consistent with the different behaviour of these kinds of alcohols. First, the trifluoroethoxide anion $3 \mathrm{f}$ is not able to deprotonate glycidol (the equilibrium is largely favourable to $3 \mathrm{f}$ by $8.2 \mathrm{kcal} \mathrm{mol}^{-1}$ ). This results in lower competition of the glycidol dimerization reaction (although the uncatalyzed dimerization still could play a small role), and hence more selectivity toward the target compound, [3F.0.0] (8c). In fact, although the glycidol dimerization reaction is still intrinsically favoured over the epoxide aperture with $3 \mathbf{f}$ ( $21.7 \mathrm{vs.} 23.5 \mathrm{kcal} \mathrm{mol}^{-1}$ ), taking into account the previous glycidol deprotonation equilibrium the situation completely reverses ( 29.9 vs. $23.5 \mathrm{kcal} \mathrm{mol}^{-1}$ ), making cycle I favourable over cycle IV by $\left.6.4 \mathrm{kcal} \mathrm{mol}^{-1}\right)$. Note that the activation barrier for the reaction of $\mathbf{3} \mathbf{f}$ with $\mathbf{1}$ is higher than any of those calculated for $\mathbf{3 a}$ and $\mathbf{3 c}$, in agreement with the 
observed relative reactivity of the three alcohols. Of course, the resulting $\mathbf{5 f}$ and $\mathbf{7 f}$ intermediates may still react with an additional glycidol molecule, leading to the $11 \mathrm{f}$ and $\mathbf{1 4 f}$ byproducts, and thus decreasing the global selectivity of the reaction to the glycerol monoether $\mathbf{8 f}$. However, in this case the protonation of these intermediates to lead $\mathbf{8 f}$ is thermodynamically favoured, so these lateral reactions should play a minor role. The kinetic simulations in this case lead to selectivities over $99 \%$ both with dropwise and at once addition of glycidol, again in line with the behaviour experimentally observed.

\section{Experimental}

\section{Computational methods}

Quantum mechanical calculations were carried out by using the Gaussian 09 package. ${ }^{53}$ All the geometry optimizations, energies, vibrational frequency calculations were performed at the B3LYP/6-311++G $(d, p)$ theoretical level. The use of diffuse functions on all atoms is essential to assure a correct description of the anionic species. This level of theory has proven to yield accurate activation parameters and geometries. Nevertheless, the consistency of this approach has been tested by using seven other functionals to calculate the activation energy of the reaction between the methoxide anion and glycidol (see Supporting Information for details). All stationary points were characterized by harmonic analysis. In particular, transition structures were characterized by the presence of only one imaginary frequency, corresponding to the atomic movement along the reaction coordinate. Unless otherwise stated, all Gibbs free energies reported in this paper correspond to calculations at room temperature. Some frequency calculations were also carried out at $65^{\circ} \mathrm{C}$ (the temperature experimentally used in the reactions). Although the free energy activation barriers increase by ca. $1.3 \mathrm{kcal} \mathrm{mol}^{-1}$ with regard to the room temperature values, the relative activation barriers (responsible for reaction selectivity) remain almost identical. Solvent effects in highly polar media, such as alcohols, are quite important in the reactions studied. To take into account these effects, al calculations were carried out using the polarized-continuum model (PCM) with the Gaussian 09 internally stored parameters for methanol, 1-butanol and 2,2,2-trifluoroethanol.

\section{General}

Gas chromatography was carried out in a Hewlett Packard 7890 series II Gas Chromatograph using a column of phenyl silicone $5.5 \%$ (Zebron ZB-5HT Inferno $30 \mathrm{~m} \times 0.25 \mathrm{~mm} \times 0.25$ ? $\mathrm{m}$ ) Helium as carrier gas, and equipped with a flame ionization detector (FID).

${ }^{1} \mathrm{H}-,{ }^{13} \mathrm{C}-$, and ${ }^{19} \mathrm{~F}-\mathrm{NMR}$ spectra (DMSO- $\mathrm{d}_{6}, \delta \mathrm{ppm}, \mathrm{J} \mathrm{Hz}$ ) were obtained using a Bruker AV-400 instrument with TMS as standard. MS spectra were obtained using a Bruker MicroTof- $\mathrm{Q}$ spectrometer with electrospray ionization. Boiling points were determined using differential scanning calorimetric (DSC) analysis in a TA Instruments DSC-Q20, calibrated with indium, using micropore aluminium pans, in a range of 298-673 K, at atmospheric pressure. Boiling points were determined using onset temperature.

Glycidol, diglyme, 1,5,7-Triazabicyclo[4.4.0]dec-5-ene (TBD) TBD-PS, TBD-silica, magnesium oxide, anisole and lithium hydroxide were purchased from Sigma-Aldrich. Methanol, ethanol, isopropanol, potassium hydroxide and sodium hydroxide were purchased from Scharlab. Butanol, 2,2,2 trifluoroethanol, 2-ethylhexan-1-ol, phenol and strontium hydroxide were purchased from Alfa Aesar. Basic resin Amberlyst ${ }^{\circledR}$ IRA-400 was purchased from Carlo Erba. All the alcohols were dried and distilled over calcium hydride prior to use. The metal hydroxides purity was determined by titration using potassium hydrogen phtalate as titrating agent.

\section{Synthesis of glycerol monoethers [R.0.0]}

The appropriate amount of alcohol $(1500 \% \mathrm{~mol}$ with respect to glycidol), the catalyst $(10,20$ or $30 \% \mathrm{~mol}$ with respect to glycidol), and diglyme or anisole as internal standard (15\% w/w with respect to glycidol) were placed into a round bottomed flask. The reaction was stirred and heated at the desired temperature $\left(65,45\right.$, or $\left.25^{\circ} \mathrm{C}\right)$ under argon until total dissolution of the catalyst. Then, glycidol $(4.35 \mathrm{mmol})$ was added dropwise for $15 \mathrm{~min}$. The reaction was monitored at different times by extracting samples that were neutralized with $\mathrm{HCl} 0.3 \mathrm{M}$ previous to injection in GC. After total consumption of glycidol, in the case of using homogeneous catalysts, the reaction was quenched with $\mathrm{HCl} 0.3 \mathrm{M}$, whereas in the case of heterogeneous catalysts the solid was just filtered off. Conversions and yields were determined by GC and checked by ${ }^{1} \mathrm{H}-\mathrm{NMR}$ at the end of reaction. Reactions were scaled up in order to obtain ca. $100 \mathrm{~g}$ of product. After reaction work-up the corresponding glycerol monoether was purified by vacuum distillation.

\section{Glycerol monoethers characterization}

All the products were identified and characterized by ${ }^{1} \mathrm{H}-,{ }^{13} \mathrm{C}-$, and ${ }^{19} \mathrm{~F}-\mathrm{NMR}$, and by HRMS. Boiling points were determined by DSC measurements.

More experimental details and full characterization of products are gathered in the Supporting Information.

\section{Conclusions}

A simple and green reaction scheme has been investigated for the synthesis of glycerol monoethers from glycidol and the corresponding alcohol, catalyzed by bases, without need of either excess of glycidol or additional solvents. The influence of the nature of the alcohol, as well as of the reaction conditions, have been studied both experimentally and theoretically. The synthetic scheme developed provides fairly good results in some cases, but there are serious selectivity issues in others, due to the concurrence of side reactions. Thus, the synthesis of monoalkyl glycerol ethers can be achieved using alkaline hydroxides as catalysts and smooth reaction conditions, with stoichiometric amounts of glycidol. Good yields are obtained with methanol, but only moderate yields are obtained when increasing the length of the alkyl chain. The computational mechanistic studies highlight that the easy dimerization of glycidol in these reaction conditions is favoured when using larger alcohols, becoming the principal reaction. This situation is worsened by the higher relative acidity of glycidol with regard to longer alkyl alcohols. The slow addition of glycidol partially overcomes this drawback. The noticeable increase of selectivity when using more acidic alcohols such as phenol or trifluoroethanol (above all in the reactions catalyzed by $\mathrm{LiOH}$ ) is in line with this mechanistic view. In the best case, total glycidol conversion with $94 \%$ selectivity to the desired product can be 
achieved. Finally, in the case of methanol, homogeneous alkaline hydroxides could be replaced by a recoverable basic heterogeneous catalyst (Amberlyst ${ }^{\circledR}$ IRA 400).

\section{Acknowledgements}

The authors acknowledge financial support from MINECO (project CTQ2014-52367-R), Gobierno de Aragón (Group E11) and the European Regional Development Funds. We are indebted to the Galicia Supercomputation Center (CESGA) for computing facilities. A. Leal acknowledges the MECD for a FPU grant.

\section{Notes and references}

1 U. Biermann, U. Bornscheuer, M. A. R. Meier, J. O. Metzger, H. J. Schäfer, Angew. Chem. Int. Ed., 2011, 50, 3854-3871.

2 U. Biermann, W. Friedt, S. Lang, W. Lühs, G. Machmüller, J. O. Metzger, M. Rüsch gen. Klaas, H. J. Schäfer, M. P. Schneider, Angew. Chem. Int. Ed., 2000, 39, 2206-2224.

3 A. E. Kerenkan, F. Béland, T.-O. Do, Catal. Sci. Technol., 2016, 6, 971-987.

4 S. Miao, P. Wang, Z. Su, S. Zhang, Acta Biomater., 2014, 10, 1692-1704.

5 A. Gandini, T. M. Lacerda, A. J. F. Carvalho, E. Trovatti, Chem. Rev., 2016, 116, 1637-1669.

6 M. Besson, P. Gallezot, C. Pinel, Chem. Rev., 2014, 114, 18271870.

7 P. Gallezot, Chem. Soc. Rev., 2012, 41, 1538-1558.

8 J. O. Metzger, Eur. J. Lipid Sci. Technol., 2009, 111, 865-876.

9 J. M. Fraile, J. I. García, C. I. Herrerías, E. Pires, Synthesis, 2017, 49, 1444-1460.

10 T. Mizugaki, R. Arundhathi, T. Mitsudome, K. Jitsukawa, K. Kaneda, ACS Sustain. Chem. Eng., 2014, 2, 574-578.

11 F. Bauer, C. Hulteberg, Biofuels, Bioprod. Biorefining, 2013, 7, 43-51.

12 Z. Y. Zakaria, N. A. S. Amin, J. Linnekoski, Biomass, Bioenergy, 2013, 55, 370-385.

13 H. W. Tan, A. R. Abdul Aziz, M. K. Aroua, Renew. Sustain. Energy Rev., 2013, 27, 118-127.

14 C. A. G. Quispe, C. J. R. Coronado, J. A. Carvalho Jr., Renew. Sustain. Energy Rev., 2013, 27, 475-493.

15 C. Li, K. L. Lesnik, H. Liu, Energies, 2013, 6, 4739-4768.

16 C.-H. C. Zhou, J. N. Beltramini, Y.-X. Fan, G. Q. M. Lu, Chem. Soc. Rev., 2008, 37, 527-549.

17 F. Jérôme, Y. Pouilloux, J. Barrault, ChemSusChem, 2008, 1, 586-613.

18 A. Behr, J. Eilting, K. Irawadi, J. Leschinski, F. Lindner, Green Chem., 2008, 10, 13.

19 J. A. Kenar, Lipid Technol., 2007, 19, 249-253.

20 J. I. García, H. García-Marín, E. Pires, Green Chem., 2014, 16, 1007-1033.

21 A. E. Díaz-Álvarez, J. Francos, P. Croche, V. Cadierno, Curr. Green Chem., 2014, 1, 51-65.

22 A. Díaz-Álvarez, V. Cadierno, Appl. Sci., 2013, 3, 55-69.

23 A. Wolfson, A. Snezhko, T. Meyouhas, D. Tavor, Green Chem. Lett. Rev., 2012, 5, 7-12.

24 A. E. Díaz-Álvarez, J. Francos, B. Lastra-Barreira, P. Crochet, V. Cadierno, Chem. Commun., 2011, 47, 6208-6227.

25 Y. Gu, F. Jérôme, Green Chem., 2010, 12, 1127.

26 A. R. Trifoi, P. Ş. Agachi, T. Pap, Renew. Sustain. Energy Rev., 2016, 62, 804-814.

27 J. R. Dodson, T. d C. M. Leite, N. S. Pontes, B. Peres Pinto, C. J. A. Mota, ChemSusChem, 2014, 7, 2728-2734.
28 J. Zhu, X. Li, C. Huang, L. Chen, L. Li, Carbohydr. Polym., 2013, 94, 874-881.

29 M. O. Sonnati, S. Amigoni, E. P. Taffin de Givenchy, T. Darmanin, O. Choulet, F. Guittard, Green Chem., 2013, 15 283-306.

30 M. M. C. Feltes, D. de Oliveira, J. M. Block, J. L. Ninow, Food Bioprocess Technol., 2013, 6, 17-35.

31 J. F. Izquierdo, M. Montiel, I. Pales, P. R. Outon, M. Galan, L. Jutglar, M. Villarrubia, M. Izquierdo, M. P. Hermo, X. Ariza, Renew. Sustain. Energy Rev., 2012, 16, 6717-6724.

32 M. Sutter, E. Da Silva, N. Duguet, Y. Raoul, E. Métay, M Lemaire, Chem. Rev., 2015, 115, 8609-8651.

33 Y. Gu, A. Azzouzi, Y. Pouilloux, F. Jérôme, J. Barrault, Green Chem., 2008, 10, 164-167.

34 J. I. García, H. García-Marín, J. A. Mayoral, P. Pérez, Green Chem., 2013, 15, 2283-2293.

35 J. I. García, H. García-Marín, J. A. Mayoral, P. Pérez, Green Chem., 2010, 12, 426.

36 L. Moity, M. Durand, A. Benazzouz, C. Pierlot, V. Molinier, J.M. Aubry, Green Chem., 2012, 14, 1132.

37 L. Aldea, J. I. García, J. a. Mayoral, Dalton Trans., 2012, 41, 8285.

38 H. Garcia-Marin, J. C. van der Toorn, J. A. Mayoral, J. I. Garcia, I. W. C. E. Arends, J. Mol. Catal. A, Chem., 2011, 334, 83-88.

39 E. Perales, C. Belen Garcia, L. Lomba, L. Aldea, J. Ignacio Garcia, B. Giner, Ecotoxicol. Environ. Saf., 2016, 132, 429-434.

40 J. I. García, E. Pires, L. Aldea, L. Lomba, E. Perales, B. Giner, Green Chem., 2015, 17, 4326-4333.

41 B. S. Flowers, M. S. Mittenthal, A. H. Jenkins, D. A. Wallace, J. W. Whitley, G. P. Dennis, M. Wang, C. H. Turner, V. N. Emel'yanenko, S. P. Verevkin, J. E. Bara, ACS Sustain. Chem. Eng., 2017, 5, 911-921.

42 M. Sutter, W. Dayoub, E. Métay, Y. Raoul, M. Lemaire, Chem CatChem, 2013, 5, 2893-2904.

43 M. Sutter, W. Dayoub, E. Métay, Y. Raoul, M. Lemaire, Green Chem., 2013, 15, 786.

44 R. P. Pohanish, William Andrew Publishing, Oxford, 2012, pp. 1210-1212.

45 NTP (National Toxicology Program). 2016. Report on Carcinogens, Fourteenth Edition.; Research Triangle Park, NC: U.S. Department of Health, Human Services, Public Health Service. http://ntp.niehs.nih.gov/go/roc14/.

46 S. Berkowitz, EP0024806 (A1), 1981

47 M. De Torres, I. W. C. E. Arends, J. A. Mayoral, E. Pires, G. Jimenez-Oses, Appl. Catal. A, Gen., 2012, 425, 91-96.

48 M. Pérez-Sánchez, M. Sandoval, A. Cortés-Cabrera, H. GarcíaMarín, J. V. Sinisterra, J. I. García, M. J. Hernaiz, Green Chem., 2011, 13, 2810.

49 H. Garcia-Marin, J. C. van der Toorn, J. A. Mayoral, J. I. Garcia, I. W. C. E. Arends, Green Chem., 2009, 11, 1605-1609.

50 Z.-M. Wang, X.-L. Zhang, K. B. Sharpless, Tetrahedron Lett., 1993, 34, 2267-2270.

51 S. Shen, H. Shi, H. Sun, Int. J. Chem. Kinet., 2007, 39, 440-446.

52 A. A. Bredikhin, Z. A. Bredikhina, D. V. Zakharychev, A. V. Pashagin, Tetrahedron:Asymm., 2007, 18, 1239-1244.

53 Gaussian 09, Revision E.01, M. J. Frisch, G. W. Trucks, H. B. Schlegel, G. E. Scuseria, M. A. Robb, J. R. Cheeseman, G. Scalmani, V. Barone, G. A. Petersson, H. Nakatsuji, X. Li, M. Caricato, A. Marenich, J. Bloino, B. G. Janesko, R. Gomperts, B. Mennucci, H. P. Hratchian, J. V. Ortiz, A. F. Izmaylov, J. L. Sonnenberg, D. Williams-Young, F. Ding, F. Lipparini, F. Egidi, J. Goings, B. Peng, A. Petrone, T. Henderson, D. Ranasinghe, V. G. Zakrzewski, J. Gao, N. Rega, G. Zheng, W. Liang, M. Hada, M. Ehara, K. Toyota, R. Fukuda, J. Hasegawa, M. Ishida, T. Nakajima, Y. Honda, O. Kitao, H. Nakai, T. Vreven, K. Throssell, J. A. Montgomery, Jr., J. E. Peralta, F. Ogliaro, M. Bearpark, J. J. Heyd, E. Brothers, K. N. Kudin, V. N. Staroverov, T. Keith, R. Kobayashi, J. Normand, K. Raghavachari, A. Rendell, J. C. 
Burant, S. S. Iyengar, J. Tomasi, M. Cossi, J. M. Millam, M. Klene, C. Adamo, R. Cammi, J. W. Ochterski, R. L. Martin, K. Morokuma, O. Farkas, J. B. Foresman, and D. J. Fox, Gaussian, Inc., Wallingford CT, 2016.

54 S. Kozuch, S. Shaik, Acc. Chem. Res., 2011, 44, 101-110. 